\title{
Thermoacoustic tomography of in vivo rat brain
}

\author{
Yuan Zhao*,\$, Zihui Chi**, Lin Huang*,\$, Zhu Zheng*,\$, \\ Jinge Yang** and Huabei Jiang*, ${ }^{*}, \uparrow$ \\ ${ }^{*}$ School of Physical Electronics \\ University of Electronic Science and Technology of China \\ No. 2006, Xiyuan Ave, West Hi-Tech Zone \\ Chengdu, Sichuan 611731, P.R. China \\ ${ }^{\dagger}$ Department of Medical Engineering \\ University of South Florida \\ Tampa, FL 33620, USA \\ *Center for Information in Medicine \\ University of Electronic Science and Technology of China \\ No. 2006, Xiyuan Ave, West Hi-Tech Zone \\ Chengdu, Sichuan 611731, P.R. China \\ §yuanz91@foxmail.com \\ 『hjiang1@usf.edu
}

Received 13 February 2017

Accepted 10 May 2017

Published 19 June 2017

\begin{abstract}
We present for the first time in vivo imaging of rat brain using microwave-induced thermoacoustic tomography (TAT). The in vivo imaging of rat brain was realized through an unconventional delivery of microwave energy from the front of rat brain (while the transducer was scanned along coronal plane of the animal brain), which maximized the microwave penetration into the brain. In addition, we found that the imaging contrast was highly dependent on the direction of the electric field polarization (EFP) and that more tissue structures/compositions could be revealed when both $X$ - and $Y$-EFPs were used for TAT. The in vivo TAT images of rat brain obtained were compared with the $3.0 \mathrm{~T}$ MRI images and histological photographs, and numerous important brain anatomical structures were identified. An example of our TAT approach for imaging a foreign object embedded in a rat brain was also demonstrated. This study suggests that TAT has a great potential to be used in neuroscience studies and in noninvasive imaging of brain disorders.
\end{abstract}

Keywords: Thermoacoustic imaging; tomography; rat brain.

『Corresponding author.

This is an Open Access article published by World Scientific Publishing Company. It is distributed under the terms of the Creative Commons Attribution 4.0 (CC-BY) License. Further distribution of this work is permitted, provided the original work is properly cited. 


\section{Introduction}

Currently, some existing imaging methods used to detect and diagnose brain diseases, like CT and MRI, are time-consuming, expensive and bulky. Thus they cannot be used in remote areas, or for monitoring brain injury over a long period of time. ${ }^{1}$ Microwave-induced thermoacoustic tomography (TAT), as a compact and mobile technology, ${ }^{2,3}$ can be applied to monitor the patient continuously in real time, ${ }^{4}$ either at the bedside or in the ambulance. TAT adds a significant advantage for brain imaging compared to the existing imaging methods. Typically, in order to provide sufficient resolution for neuroscience studies, the 7.0 T or higher MRI needs to be used in small animals such as mice and rats. These imaging equipments are very costly. TAT is significantly less expensive than the current methods with similar resolution. In addition, TAT is an emerging noninvasive imaging modality capable of providing high electric contrast and high ultrasound resolution imaging of tissue..$^{5,6}$ TAT has been so far used for imaging soft tissue such as joints ${ }^{7,8}$ and breast. ${ }^{4,9-11}$ While an initial effort has been made to image brain tissue using TAT, it has been limited to ex vivo tissue ${ }^{12}$ or the boundary information of in vivo rat brain. ${ }^{13,14}$ In vivo imaging would indicate an important milestone of TAT. In this paper, we describe thermoacoustic (TA) methods that allow us to obtain in vivo structural images of rat brain for the first time. Compared with monkey, rat is a better experimental animal at an early stage of feasibility study. On the other hand, we chose rat brain as our study model as it is a widely used animal model for brain studies such as craniocerebral injury, Parkinson and stroke. ${ }^{15-17}$

\section{Materials and Methods}

\subsection{Experimental setup and imaging procedure}

Our experimental setup is schematically shown in Fig. 1(a). In this TAT system, a custom-designed pulsed microwave generator (frequency: $3.0 \mathrm{GHz}$, peak power: $70 \mathrm{~kW}$, pulse duration: $750 \mathrm{~ns}$, repetition rate: $50 \mathrm{~Hz})^{18}$ was used to produce microwave pulses through a waveguide-fed pyramidal horn antenna (aperture size: $114 \times 144 \mathrm{~mm}^{2}$ ) to irradiate the rat head from the front [Fig. 1(b), coronal scanning] or from the top [Fig. 1(c), transverse scanning]. A $5 \mathrm{MHz}$ cylindrical focusing ultrasound transducer (V326, Olympus; diameter: $10 \mathrm{~mm}$; focal length: $53 \mathrm{~mm}$ ) rotated by a step motor detected the TA signal at 180 steps with a constant scanning step of $2^{\circ}$ to cover a $2 \pi$-receiving angle. The signal detected was filtered $(-3 \mathrm{~dB}$ bandwidth: $500 \mathrm{kHz}-$ $30 \mathrm{MHz}$ ) and amplified by a low-noise pre-amplifier (Preamp2-D, US Ultratek, Inc., USA) with $60 \mathrm{~dB}$. The signal was then sampled and averaged 50 times by data acquisition card controlled by a computer. The total time for data collection was $3 \mathrm{~min}$. After a second-order Butterworth low-pass filter at $1.5 \mathrm{MHz}$
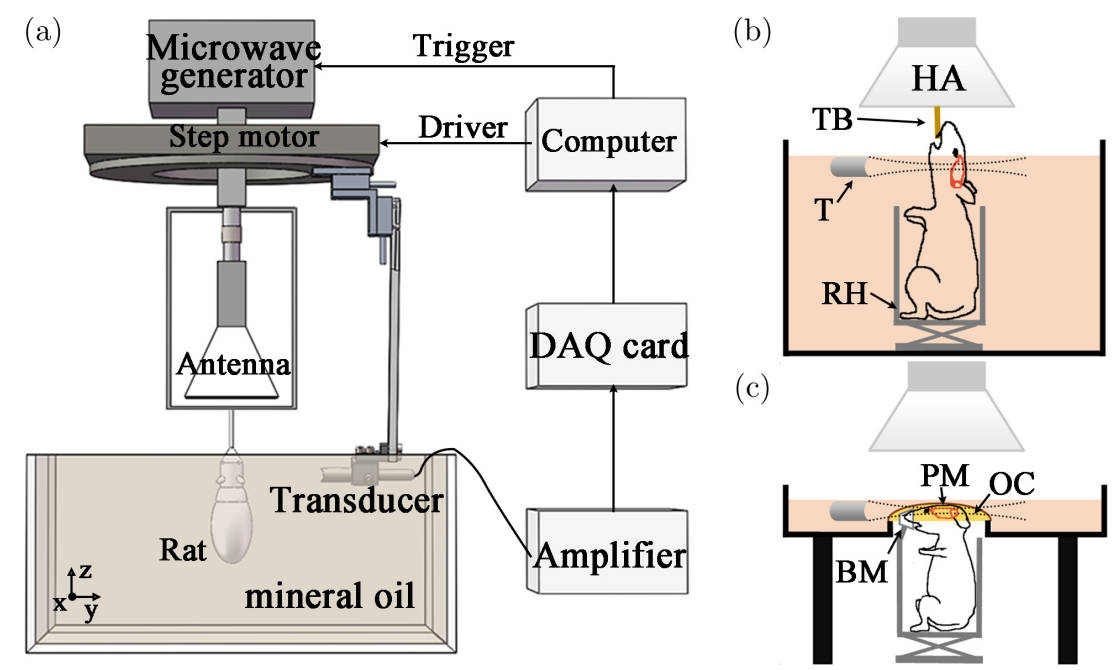

Fig. 1. (a) Schematic of TAT system for imaging of rat brain. (b) and (c) Schematics showing the coronal and transverse scanning, respectively. RH: rat holder, PM: plastic membrane, OC: oil-based couplant, BM: breathing mask, TB: tooth bar, T: transducer and HA: waveguide-fed pyramidal horn antenna. 
to suppress noises, the TAT signals were used to reconstruct TAT images using a delay and sum algorithm. ${ }^{19,20}$ The actual averaged microwave power density at the surface of rat brain is below $0.32 \mathrm{~mW} / \mathrm{cm}^{2}$, which is far below the safety standard $\left(10 \mathrm{~mW} / \mathrm{cm}^{2}\right.$ at $\left.3 \mathrm{GHz}\right) .{ }^{21}$

\subsection{TATs of different rat postures}

We first studied how different postures of rat brain influence the electric field distribution in tissue by numerical simulations and animal experiments. Both the simulations and experiments were based on the postures of rat brain depicted in Figs. 1(b) and 1(c). In our setup, the key conditions including the relative locations of the transducer, rat brain and antenna and the distance between oil surface and the rat brain were kept the same. In the experiments, for the posture of coronal scanning, as shown in Fig. 1(b), a home-made bakelite holder suspended the rat by a tooth bar. The whole body of rat except for the mouth and nose was immersed in mineral oilfilled plexiglass tank. The mouth and nose of the rat was positioned above the surface of oil so that the rat could breathe freely. The head of rat stayed vertical due to the force of gravity. For the transverse scanning, ${ }^{13,14}$ a customized holder (3D printed) was used to keep the rat head horizontal. The rat head protruded into mineral oil-filled plexiglass tank through a hole at the bottom of the tank under a piece of clear membrane. A thin layer of oil-based couplant was used to couple ultrasonic transmission between membrane and skin of rat head.
Specifically, we chose an oil-based couplant rather than the traditional ultrasonic couplant since the traditional ultrasonic couplant contains water. Water could absorb and scatter microwave and reduce signal-to-noise ratio. Furthermore, oil-based couplant has a higher coefficient of viscosity to avoid couplant rusting streaming down to the breathing mask for better rat breathing.

A finite integral time-domain method (FITD) based on the industry-standard CST 2014 software package $^{22}$ was used to simulate the distribution of power loss density for both coronal and transverse scanning. A simplified CST rat brain model was used for simulations, which consisted of muscle and brain parenchyma tissues. The elliptical cylinder representing the brain was inserted into a bigger elliptical-like cylinder [see Figs. 2(a) and 2(b)]. Brain's conductivity and permittivity are typically $2 \mathrm{~S} / \mathrm{m}$ and 48, respectively. Muscle's conductivity and permittivity are $2.1 \mathrm{~S} / \mathrm{m}$ and 52 , respectively. Oil's permittivity is $2.33 .{ }^{23}$ The numerical model setup was very similar to the experimental setup except that the rat was simplified [as shown in Fig. 1(a)]. Its energy distribution has been described in detail in our previous article. ${ }^{24}$ The results from the simulations and experiments are shown in Figs. 2(c)-2(f). For transverse scanning [Fig. 2(c)], we see that the power loss energy was mainly distributed outside the rat brain (indicated by the black ellipse) within the muscle. This was further confirmed by the animal experiment [Fig. 2(d)] where the TAT image of the brain showed very low contrast in the brain regions indicated by the

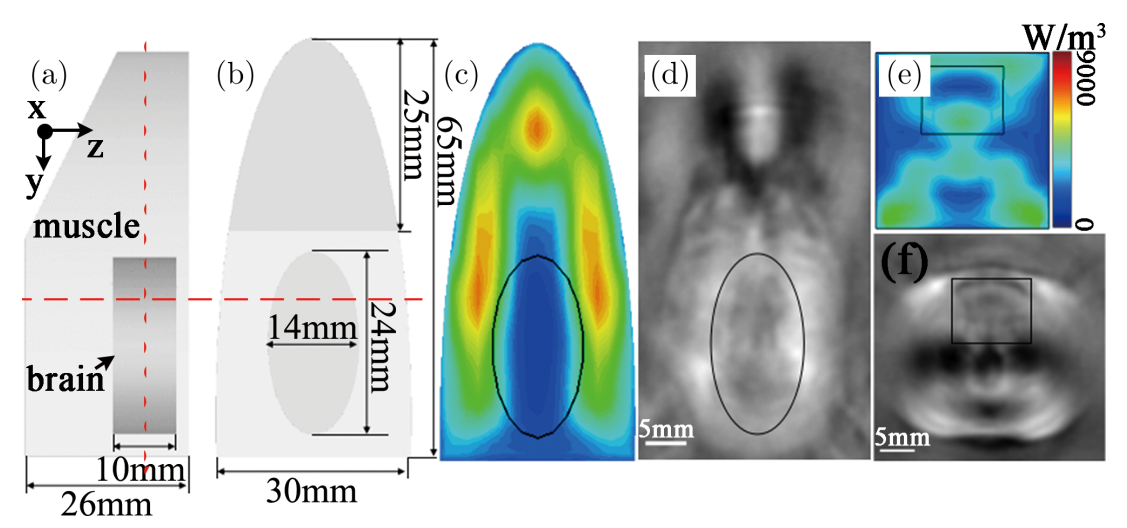

Fig. 2. (a) and (b) The left and top views of simulation model of rat head. (c) Simulated power loss density distribution along transverse section [i.e., the $x-y$ plane as shown in (a) by the short dashed line]. (d) In vivo transverse TA image of rat head corresponding to (c). (e) Simulated power loss density distribution along coronal sections [i.e., the $x-z$ plane as shown in (a) by the long dashed line]. (f) In vivo coronal TA image of rat head corresponding to (e). The brain regions indicated by black ellipse [panels (c) and (d)] and black rectangle [panels (e) and (f)] correspond to the elliptical cylinder in the simulation model in (a) and (b). 
ellipse. For coronal scanning, while reduced power loss density was seen in some regions of the brain, we can see that significant amount of energy penetrated into the brain regions [Fig. 2(e), indicated by the black rectangle]. This was again consistent with the experimental result [Fig. 2(f)]. Assuming the input power of horn antenna as $1 \mathrm{~W}$ in simulations, the total power loss density values in the brain were calculated to be $3.72 \mathrm{~mW}$ and $5.02 \mathrm{~mW}$ for transverse and coronal scanning, respectively. This indicates that more than $35 \%$ energy was delivered into the brain when coronal scanning was used, compared to transverse scanning.

\subsection{Image processing method based on electric field polarization}

The differences of conductivity and permittivity ${ }^{25}$ among tissues of rat head and a difference between oil $(\varepsilon \sim 2.33)$ and skin $(\varepsilon \sim 37-42)$ can lead to nonuniform distribution of electric field in rat head. Orientation of the object relative to incident EFP will significantly impact TA signal generated. So thermoacoustic image contrast is dependent on the EFP. ${ }^{26-29}$ In this work, in order to utilize complementary information on two images from the two EFPs while retaining the independence of the single image, we propose to use a new image fusion method. As shown in Fig. 1(a), we rotated the horn antenna independently by an adjustable coaxial connector to achieve polarization change, while keeping the rat quiescent at the same time. In this way, we could get two TAT images from $X$-EFP [Fig. 3(a)] and $Y$-EFP [Fig. 3(b)], respectively. We used Figs. 3(a) and 3(b) to get a fused image of rat brain [Fig. 3(c)]. Figure 3(d) was a segmented and annotated image from Fig. 3(c). The first step in the processing of fusion was to normalize the TAT images from $X$ - and $Y$-EFPs between 0 and 1 , respectively. The second step was to build an RGB color image, where red channel value and green channel value were written from normalized TAT images for $X$-and $Y$-EFPs, respectively. The blue channel value was set to 0 . The colormap is shown in Fig. 3(e). Figure 3(f) shows the corresponding brain histological photograph after the TAT imaging.

\subsection{Animal preparation}

Six adult Sprague-Dawley rats having a weight from $200 \mathrm{~g}$ to $400 \mathrm{~g}$ were imaged by TAT. One rat

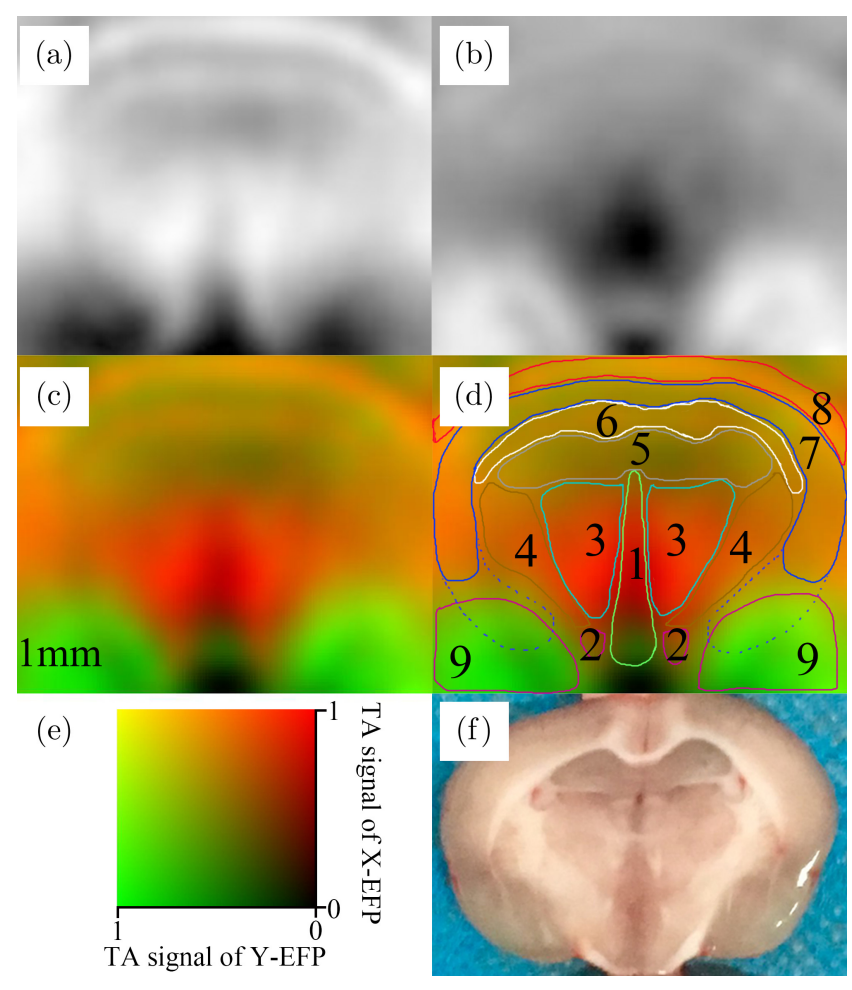

Fig. 3. TAT images of (a) $X$ - and (b) $Y$-EFPs were used to obtain a fused image (c) of rat brain $(n=3)$ in vivo. (d) Segmented and annotated image from (c): 1: Cerebral longitudinal fissure, 2: spinal trigeminal tract, 3: striatum, 4: fiber tracts/ white matter, 5: hippocampal formation, 6: corpus callosum and associated subcortical white matter, 7: neocortex, 8: skin and 9: middle ear. (e) Colormap of fusion images. (f) Corresponding histological photograph obtained after TAT imaging.

was used for horizontal posture. Three rats were used for coronal posture with two electric field polarization directions, while one of them was also used for foreign object brain imaging experiment. Two rats were used for coronal posture with only X-EFP. All rats were anesthetized using chloral hydrate $(10 \%$ concentration, $0.04 \mathrm{~mL} / 10 \mathrm{~g})$ during TAT and MRI. The hairs were shaved and removed with depilatory creams.

\subsection{In vivo $M R I$}

In order to cross-validate our TAT results, T2*weighted gradient-echo MRI of rat was acquired on a 3.0 T MRI scanner (GE Discovery MR750) at the MRI Research Centre of UESTC half an hour before TAI. MRI data were acquired with an animal coil [repetition time $[\mathrm{TR}]=2000 \mathrm{~ms}$, echo time $[\mathrm{TE}]=55.92 \mathrm{~ms}$, flap angle $[\mathrm{FA}]=90^{\circ}$, matrix $=$ $128 \times 128$, field of view $[\mathrm{FOV}]=10 \times 10 \mathrm{~cm}^{2}$, slice 
thickness/gap $=2 \mathrm{~mm} / 1 \mathrm{~mm}$, number of averages $=4$ and total image time is $2 \min 49 \mathrm{~s}$ for a rat].

\section{Results and Discussion}

\subsection{Electric field polarization on imaging contrast}

We found that TAT imaging contrast is highly dependent on the direction of EFP from the horn antenna, i.e., some tissue structures/compositions are revealed using one EFP direction, while other tissue structures/compositions are imaged by another EFP direction. Images of $X$-EFP [Fig. 3(a)] and $Y$-EFP [Fig. 3(b)] were used to obtain a fused image [Fig. 3(c), colormap shown in Fig. 3(e)] of rat brain $(n=3)$. To better illustrate different regions, we segmented Fig. 3(c) based on the amplitude of TAT images and labeled different segmentations [Fig. 3(d)] according to a stereotaxic atlas of rat brain. ${ }^{30}$ Histological photograph of brain slice [Fig. 3(f), obtained after the TAT imaging] confirmed the TAT results, a number of important tissue structures [Fig. 3(d)] are identified including cerebral longitudinal fissure, spinal trigeminal tract, striatum, fiber tracts/white matter, hippocampal formation, corpus callosum and associated subcortical white matter, neocortex, skin, the middle ear and inner ear. We found that the major white matter structures in thermoacoustic image agreed well with corresponding MRI segmented images. ${ }^{31}$
We even could distinguish between gray and white matters in some brain regions. For example, in Fig. 3(d), corpus callosum (no. 6, white matter) could be distinguished from neocortex (no. 7, grey matter) and hippocampal formation (no. 5, gray matter). Moreover, we obtained MRI images of a rat brain and conducted TAT imaging of the same rat brain (see Fig. 4 for results). We found that the TAT images are comparable to the MRI images to quite some extent and that numerous tissue structures seen on MRI are also revealed on TAT, including trachea, palate bone, mandible, tongue, temporal bone, striatum, neocortex, larynx, scalp, middle ear, zygomar and gland. ${ }^{32}$ These results suggest that TAT has a great potential to be used in neuroscience studies. The spatial resolution of this system is estimated to be about $0.9 \mathrm{~mm}$ (Ref. 33) due to limitation by the pulse width of the microwave generator. The resolution is an important improvement that needs to be undertaken by using a shorter-width nanosecond microwave pulse generator in the future. For instance, the spatial resolution of $45-105 \mu \mathrm{m}$ can be achieved by a $10 \mathrm{~ns}$ microwave pulse generator. ${ }^{6,34}$

\subsection{Foreign object detection}

To demonstrate the potential of our TAT approach for detecting an abnormality in brain, we inserted a soybean sauce-filled plastic tube ( $3 \mathrm{~mm}$ in diameter) into the striatum region of a rat brain. Figure 5(a)

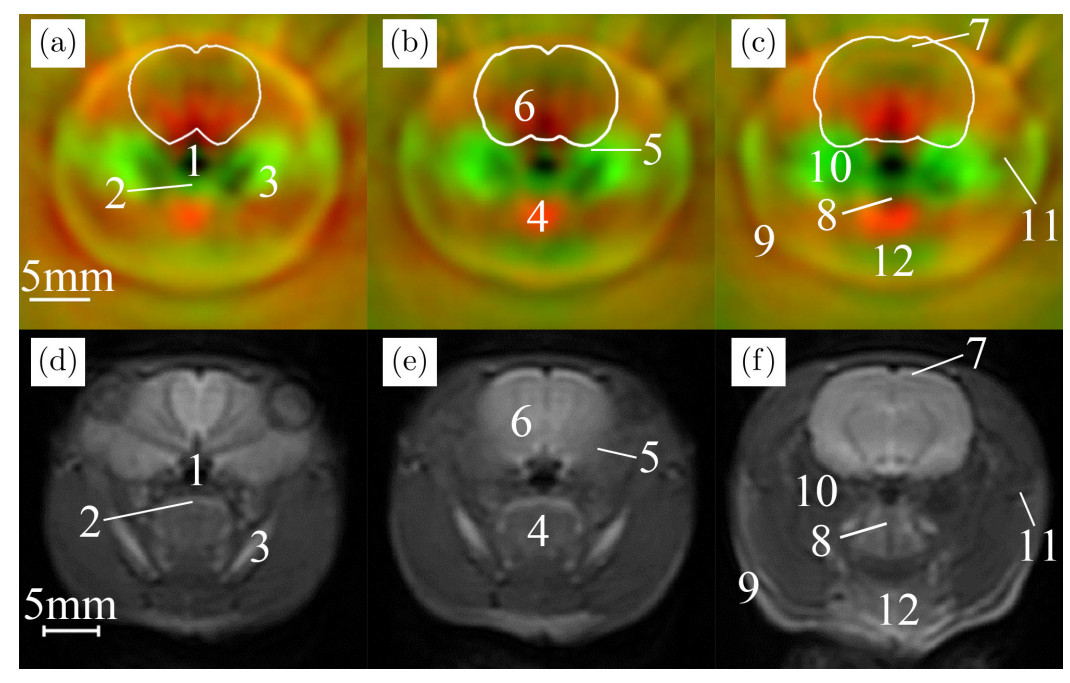

Fig. 4. In vivo TAT [panels (a)-(c)] and $3 \mathrm{~T} \mathrm{T2*-MRI} \mathrm{[panels} \mathrm{(d)-(f)]} \mathrm{images} \mathrm{of} \mathrm{rat} \mathrm{brain}(n=3)$ at different coronal sections. Anatomical structures are identified in these images according to sectional anatomical atlas of Sprague-Dawley rat ${ }^{32}: 1:$ trachea, 2: palate bone, 3: mandible, 4: tongue, 5: temporal bone, 6: striatum, 7: neocortex, 8: larynx, 9: scalp, 10: middle ear, 11: zygomar and 12: gland. 


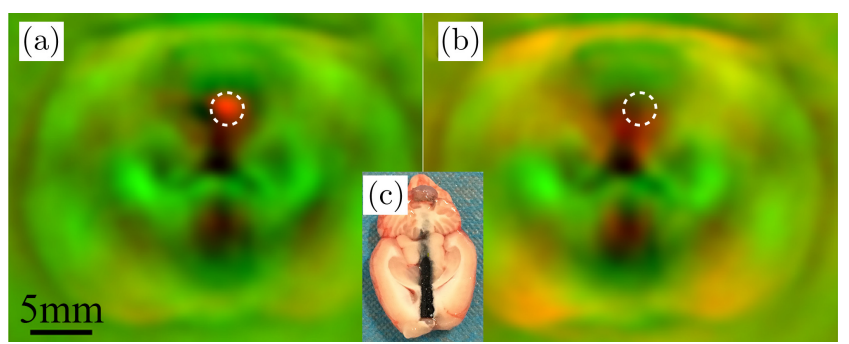

Fig. 5. (a) TAT images of foreign object-containing rat brain and (b) the same rat brain without the foreign object. Dashed circles in (a) represent the edge of foreign object determined by full width at half maximum. Dashed circles in (b) represent the same edges shown in (a). (c) Histological photograph of foreign object-containing rat brain obtained after the TAT imaging.

presents the TAT image of foreign object-containing rat brain in comparison with that for the rat brain before the foreign object was inserted shown in Fig. 5(b). We see that foreign object is clearly detected and that the recovered shape and size of the object are accurate relative to the actual shape and size of the object [see Fig. 5(c)]. TAT images were positively associated with the microwave absorbing information of various tissues. The fused image included both microwave absorbing information from the two EFPs according to the colormap shown in Fig. 3(e). In addition, we can easily compare the influence extent of different EFPs on TAT. For example, we can tell that the plastic tube's signal was excited primarily along $X$-EFP since the area of plastic tube was bright red in Fig. 5(a).

\subsection{Noise suppression}

Electrical noise and ultrasonic noise from motor may create interference through a cantilever, which directly connects with the transducer. Our system used isolated layers, made of polyamide, between the transducer and the cantilever to isolate the electric and ultrasonic noises. Because, polyamide is an insulator and can isolate the electrical interference noise produced by the motor. Furthermore, its acoustic impedance $\left(\sim 2 \mathrm{~kg} / \mathrm{m}^{2} \cdot \mathrm{s} \times 106\right.$; see Ref. 35$)$ is much less than that of the stainless steel cantilever $\left(45.40 \mathrm{~kg} / \mathrm{m}^{2} \cdot \mathrm{s} \times 106\right) .{ }^{35}$ According to Snell's law, the reflection coefficient of the interface is

$$
R=\left(\frac{Z_{2}-Z_{1}}{Z_{2}+Z_{1}}\right)^{2}=\left(\frac{45.4-2}{45.4+2}\right)^{2}=84 \% .
$$

Here, $R, Z_{1}$ and $Z_{2}$ represent the reflection coefficient, characteristic acoustic impedance of polyamide and characteristic acoustic impedance of stainless steel, respectively. Most of the noises can be significantly reduced.

\subsection{Transducers}

Since the pulse width of the TA imaging is only $0.75 \mu \mathrm{s}$, the maximum TA frequency that can be generated is roughly $1.36 \mathrm{MHz}$. We used a low-pass filter at $1.5 \mathrm{MHz}$ to eliminate the interference of the high-frequency noise. As suggested, $1.5 \mathrm{MHz}$ transducer would be more suitable for the $0.75 \mu \mathrm{s}$ pulse width microwave-generated TA signal collection. However, at the time of this study, $5 \mathrm{MHz}$ cylindrical focusing ultrasound transducer was the only one available. We are currently developing a $1.5 \mathrm{MHz}$ transducer array-based TA system to realize real-time imaging in the near future.

\section{Conclusions}

In sum, we have presented a study that allowed us to obtain TAT images of rat brain in vivo for the first time, to our knowledge. This became possible because of the use of coronal scanning and full consideration of electric field polarization on imaging contrast. We plan to apply the approaches described in this work to next-step in vivo experiments for studying brain diseases such as stroke in the near future.

\section{Acknowledgments}

This research was supported in part by the 1000Talent Plan via the University of Electronic Science and Technology of China, Chengdu.

\section{References}

1. A. T. Mobashsher, 2016.

2. Z. Ji, W. Ding, F. Ye, C. Lou, Ultrason. Imaging 38(4), 276 (2016).

3. G. Alexopoulos, K. C. Boyle, N. Dolatsha, N. Hao, B. T. Khuri-Yakub, A. Arbabian, Proc. IEEE Mtt-S Int. Microwave Symp. (2016).

4. F. Ye, Z. Ji, W. Ding, C. Lou, S. Yang, D. Xing, IEEE Trans. Med. Imaging 35(3), 839-844 (2016).

5. R. A. Kruger, K. D. Miller, H. E. Reynolds, W. L. Kiser, Jr., D. R. Reinecke, G. A. Kruger, Radiology 216(1), 279-283 (2000).

6. C. Lou, S. Yang, Z. Ji, Q. Chen, D. Xing, Phys. Rev. Lett. 109(21), 218101 (2012). 
7. H. Lin, Q. Weizhi, Z. Yuan, C. Zihui, Z. Nengzhu, R. Jian, L. Dakun, $X$-Acoust., Imag. Sens. 1(1) (2015).

8. Z. Chi, Y. Zhao, L. Huang, Z. Zheng, H. Jiang, Med. Phys. 43(12), 6226 (2016).

9. L. Huang, J. Rong, L. Yao, W. Z. Qi, D. Wu, J. Y. Xu, H. B. Jiang, Chin. Phys. Lett. 30(12), 64-65 (2013).

10. Z. Ji, C. Lou, S. Yang, D. Xing, Med. Phys. 39(11), 6738-6744 (2012).

11. Z. Ji, W. Ding, F. Ye, C. Lou, D. Xing, Appl. Phys. Lett. 107(9), 094104 (2015).

12. Y. Xu, L. V. Wang, IEEE Trans. Ultrason. Ferroelectr. Freq. Control 53(3), 542-548 (2006).

13. S. Yang, X. Da, X. Liang-Zhong, Sci. China Ser. G, Phys. Mech. Astron. (S1), 101-109 (2007).

14. L. Nie, H. Guo, Proc. SPIE 7280 (2008).

15. S. Chen, C. Hsu, E. Hogan, H. Maricq, J. Balentine, Stroke 17(4), 738-743 (1986).

16. C. E. Dixon, S.-J. Liu, L. W. Jenkins, M. Bhattachargee, J. S. Whitson, K. Yang, R. L. Hayes, Behav. Brain Res. 70(2), 125-131 (1995).

17. L. M. Björklund, R. Sánchez-Pernaute, S. Chung, T. Andersson, I. Y. C. Chen, K. S. P. McNaught, A.-L. Brownell, B. G. Jenkins, C. Wahlestedt, K.-S. Kim, Proc. Natl. Acad. Sci. 99(4), 2344-2349 (2002).

18. L. Huang, L. Yao, L. X. Liu, J. Rong, H. B. Jiang, Appl. Phys. Lett. 101(24), 244106 (2012).

19. C. G. Hoelen, F. F. de Mul, Appl. Opt. 39(31), 58725883 (2000).

20. L. Xi, H. Jiang, Appl. Phys. Lett. 107(6), 063701 (2015).

21. L. Huang, W. Cai, Y. Zhao, D. Wu, L. Wang, Y. Wang, D. Lai, J. Rong, F. Gao, H. Jiang, J. Innov. Opt. Health Sci. 09(05) (2015).

22. A. Hajiaboli, S. Kellnberger, V. Ntziachristos, D. Razansky, Prog. Electromagn. Res. 139(3), 105119 (2013).
23. S. Gabriel, R. W. Lau, C. Gabriel, Phys. Med. Biol. 41(11), 2271-2293 (1996).

24. X. Bi, L. Huang, J. S. Du, W. Z. Qi, Y. Gao, J. Rong, H. B. Jiang, Acta Phys. Sin. 64(1), 14611473 (2015) (in Chinese).

25. S. Gabriel, R. W. Lau, C. Gabriel, Phys. Med. Biol. 41(11), 2251-2269 (1996).

26. S. P. Delrio, R. A. Kruger, R. B. Lam, D. R. Reinecke, Proc. SPIE 7564, 75642D (2010).

27. S. K. Patch, L. P. Yan, Proc. SPIE 7177(17), 717721 (2009).

28. C. H. Li, G. Ku, P. Manojit, L. H. V. Wang, Proc. Soc. Photo. Opt. Insrum. Eng. 6856(2-3), 85612 (2008).

29. C. Li, M. Pramanik, G. Ku, L. V. Wang, Phys. Rev. E, Stat. Nonlinear Soft Matter Phys. 77, 031923 (2008).

30. G. Paxinos and C. Watson, The Rat Brain in Stereotaxic Coordinates, Academic Press (2007).

31. P. A. Valdés-Hernández, A. Sumiyoshi, H. Nonaka, R. Haga, E. Aubert-Vásquez, T. Ogawa, Y. IturriaMedina, J. J. Riera, R. Kawashima, Front. Neuroinform. 5, 26 (2011).

32. L. Qian, G. Hui, Sectional Anatomical Atlas of Sprague-Dawley Rat, China Press (2010).

33. L. Huang, T. Li, H. Jiang, Med. Phys. 44 (2017).

34. M. Omar, S. Kellnberger, G. Sergiadis, D. Razansky, V. Ntziachristos, Med. Phys. 39(7), 4460-4466 (2012).

35. P. O. Moore (Ed.), Nondestructive Testing Handbook: Ultrasonic Testing, Third Edition, Vol. 7, ASNT (2007). 